Ciência Florestal, Santa Maria, v. 23, n. 3, p. 291-304, jul.-set., 2013

ISSN 0103-9954

\title{
GERMINAÇÃO DE SEMENTES E EMERGÊNCIA DE PLÂNTULAS DE Anadenanthera colubrina (Vell.) Brenan var. cebil (Griseb.) Altschut, FABACEAE, ESTABELECIDA EM FRAGMENTOS FLORESTAIS DO CERRADO, MG
}

\author{
SEED GERMINATION AND SEEDLING EMERGENCE OF Anadenanthera colubrina (Vell.) Brenan \\ var. cebil (Griseb.) Altschut, FABACEAE, ESTABLISHED IN FOREST FRAGMENTS OF CERRADO, \\ MINAS GERAIS STATE
}

\author{
Marieta Caixeta Dorneles ${ }^{1}$ Marli A. Ranal ${ }^{2}$ Denise Garcia de Santana ${ }^{3}$
}

\begin{abstract}
RESUMO
A estratégia de germinação dos diásporos é fundamental para a sobrevivência das espécies, sendo um dos fatores que permitem a sua manutenção e regeneração no ambiente. A germinação das sementes e a emergência das plântulas de Anadenanthera colubrina foram estudadas para analisar a eficiência reprodutiva da espécie, oriunda de fragmentos de vegetação do bioma Cerrado. Os frutos foram coletados no Vale do Rio Araguari, MG. O teor de água a $105^{\circ} \mathrm{C}$, a germinação em condições controladas de laboratório e a emergência das plântulas em estufa semiaberta, coberta com sombrite, foram analisados. As sementes apresentaram baixo teor de água, entre 6,7 e $10,7 \%$. A germinabilidade e a porcentagem de emergência foram altas para quase todos os indivíduos estudados, estando entre 68 (indivíduo 2) e 85 - 94\% (para os demais indivíduos); 38 (indivíduo 2) e 78 - 91\% (para os demais), respectivamente. A velocidade dos processos foi alta, com o término da germinação dentro de 12,8 dias e emergência em 18,8 dias. Os processos foram heterogêneos, com valores do coeficiente de variação do tempo acima de 28,5\%; assíncrono, sendo registrados valores acima de 1,5 bits para a incerteza e abaixo de 0,3812 para a sincronia. As sementes desta espécie se mostraram eficientes em ambos os processos e a heterogeneidade e assincronia podem ser indicativos dos efeitos das oscilações ambientais durante a formação das sementes, além da variabilidade genética, intrínseca de cada indivíduo da população. A rapidez e o alto potencial de germinação das sementes desta espécie também podem viabilizar programas de revegetação, com a produção de grande quantidade de plantas jovens em curto tempo.
\end{abstract}

Palavras-chave: angico-branco; espécie florestal; medidas de emergência; medidas de germinação.

\begin{abstract}
The seed germination strategy is important for the survival of species, allowing their maintenance and regeneration in the environment. Seed germination and seedling emergence of Anadenanthera colubrina were studied to examine the reproductive efficiency of this species, typical of Cerrado biome. The fruits were collected in 'Vale do Rio Araguari', in MG state. The water content at $105{ }^{\circ} \mathrm{C}$, the germination in controlled laboratory conditions and seedling emergence in semi-open greenhouse were analyzed. The seeds had low water content, from 6.7 to $10.7 \%$. The germinating ability and the emergence percentage were high for almost all studied individuals, being registered 68 for seeds of individual number 2 and 85 - 94\% for the others; $38 \%$ (individual number 2) and 78 - 91\% (for the others), respectively. The speed of the processes was high, with the end of germination within 12.8 days and emergence within 18.8 days. The processes were heterogeneous, with values of coefficient of variation of the germination or emergence time above $28.5 \%$; asynchronous, with values of uncertainty above 1.5 bits and synchrony below 0.3812 . The

1 Bióloga, Dra., Programa de Pós-graduação em Ecologia e Conservação de Recursos Naturais, Universidade Federal de Uberlândia, Campus Umuarama, Bloco 2D, Caixa Postal 593, CEP 38400-902, Uberlândia (MG). Bolsista do CNPQ.marietacdorneles@hotmail.com

2 Bióloga, Dra., Professora Titular do Instituto de Biologia, Universidade Federal de Uberlândia, Campus Umuarama, Bloco 2D, Caixa Postal 593, CEP 38400-902, Uberlândia (MG).ranal@ufu.br

3 Engenheira Agrônoma, Dra., Pesquisadora CNPq nível 2, Professora Adjunta do Instituto de Ciências Agrárias, Universidade Federal de Uberlândia, Campus Umuarama, Bloco 4C, Caixa Postal 593, CEP 38400-902, Uberlândia (MG).dgsantana@umuarama.ufu.br

Recebido para publicação em 12/07/2011 e aceito em 5/04/2012
\end{abstract}

Ci. Fl., v. 23, n. 3, jul.-set., 2013 
seeds of this species were efficient in both processes and the heterogeneity and asynchrony may indicate the effects of environmental fluctuations during the seed formation, besides the genetic variability, intrinsic to each individual of the population. The speed and the high potential of seed germination of this species may also facilitate the programs of reforestation with the production of large numbers of seedlings in short time.

Keywords: Anadenanthera colubrina; emergence measurements; forest species; germination measurements.

\section{INTRODUÇÃO}

O desmatamento que levou à fragmentação dos hábitats naturais em todo o mundo tem contribuído para as mudanças climáticas e o desequilíbrio de muitas espécies. Espécies de ambiente fragmentado podem ser mais vulneráveis a essas modificações climáticas, uma vez que as populações estabelecidas isoladamente têm seu fluxo gênico reduzido (DURIGAN, 2003). Disso resulta diminuição da variabilidade genética que pode proporcionar a exclusão das espécies do ambiente degradado, facilitando a entrada de novos colonizadores (GASCON et al., 1999). A fragmentação também pode tornar as espécies vulneráveis ao efeito de borda, mesmo as que conservam maior variabilidade genética, sendo estas comprometidas em função do aumento da distância de dispersão, o que dificulta a sua sobrevivência e a capacidade de recolonização (GASCON et al., 1999; ROY e BLOIS, 2006). Entretanto, em fragmentos que se tornaram reservas ecológicas, esses efeitos estão sendo minimizados e a regeneração das espécies tem ocorrido com sucesso (SIQUEIRA et al., 2009; VALE et al., 2009). Isto sugere que a persistência das espécies nesses ambientes depende do seu esforço reprodutivo, de sua habilidade de competição e de sua plasticidade para superar as mudanças ambientais (KOLB e DIEKMANN, 2005).

No Bioma Cerrado, as pressões antrópicas que causaram erosão nos solos, poluição aquífera e fragmentação nas fitofisionomias proporcionaram fragilidade na biodiversidade local (KLINK e MACHADO, 2005). As 12.423 espécies vegetais que se encontram estabelecidas nesse bioma (MENDONÇA et al., 2008) estão distribuídas, em sua maioria, em $20 \%$ dos fragmentos restantes ou em 2,06\% das áreas protegidas, que estão inseridas na área original coberta por vegetação de Cerrado, que era de $1.783 .200 \mathrm{~km}^{2}$ (ALHO, 2005). Em razão disso, pesquisas com enfoque botânico e ecológico que visam conhecer o comportamento das espécies estabelecidas nos ambientes fragmentados são prioritários para que se possa observar sua resiliência.

Mudanças ambientais catastróficas propor- cionam impacto instantâneo na biodiversidade local, enquanto as transformações contínuas ao longo de um tempo podem, paulatinamente, conduzir as espécies à vulnerabilidade, o que eventualmente pode ameaçá-las de extinção (DOBSON et al., 1997). No entanto, sob pressão ambiental, as espécies podem ser selecionadas à medida que mantenham ou desenvolvam características apropriadas para a dispersão e sobrevivência (KOLB e DIEKMANN, 2005; ROY e BLOIS, 2006).

A relação entre o ambiente e a qualidade fisiológica dos diásporos tem sido estudada para espécies de diferentes biomas como forma de entender o processo de estabelecimento das populações e sua distribuição geográfica. Pesquisas com enfoque nas características morfofisiológicas e ecológicas dos diásporos permitem compreender o processo de germinação das espécies nativas (GARWOOD, 1983; CAMARGO et al., 2002; SALOMÃO, 2002; PAZ e MARTÍNEZ-RAMOS, 2003; SALOMÃO et al., 2003; SAUTU et al., 2006; FERREIRA, 2007; LIMA et al., 2008; DORNELES, 2010) e retratam seu esforço reprodutivo para se manter em fragmentos florestais (COMMANDER et al., 2009). Além disso, estudos que visam conhecer o comportamento dos diásporos durante a germinação, podem mostrar a heterogeneidade entre as unidades produzidas por uma mesma planta e entre indivíduos de uma mesma área (BERGER, 2007; FERREIRA, 2007; DORNELES, 2010). Essa heterogeneidade pode ser decorrente das condições do ambiente durante o período de formação dos diásporos, associadas às características genéticas da espécie (BASKIN e BASKIN, 1998; VEASEY et al., 2000).

Anadenanthera colubrina (Vell.) Brenan var. cebil (Griseb.) Altschut é uma das espécies que se encontra estabelecida em fragmentos de distintas fitofisionomias do Cerrado e em outros biomas brasileiros, principalmente em floresta estacional decidual (NASCIMENTO et al., 2004) e semidecidual (CARDOSO e SCHIAVINI, 2002; PAULA et al., 2004), floresta de galeria (DIETZSCH et al., 2006), cerradão (CARVALHO, 2003), na caatinga arbórea (PEGADO et al., 2006) e nas partes secas calcárias do Pantanal (POTT et al., 2009). Esta espécie geral- 
mente apresenta alta produção anual de sementes, dispersas por autocoria entre agosto e setembro, final do período seco, e tem expressiva regeneração por germinação, estando classificada no grupo sucessional das secundárias iniciais (CARVALHO, 2003).

A ampla distribuição de Anadenanthera colubrina nas fitosionomias do Cerrado, o alto potencial de germinação de suas sementes (REGO et al., 2007; LIMA et al., 2008) e a rapidez desse processo (BARBOSA, 2003) podem favorecer o estabelecimento dessa espécie em áreas de conservação (SILVA et al., 2003), de regeneração natural (WERNECK et al., 2000; LOPES et al., 2002) e de revegetação do ambiente (LIMA et al., 2009). Entretanto, a dispersão autocórica pode limitar o seu estabelecimento, uma vez que as sementes próximas da planta-mãe estão sujeitas à maior competição e predação (DEMINICIS et al., 2009). Além disso, em fragmentos mais fragilizados, após a dispersão das sementes, o desequilíbrio ecológico dos predadores herbívoros (CARVALHO e VASCONCELOS, 1999), a invasão de gramíneas altamente competitivas e inflamáveis (KLINK e MACHADO, 2005) e o atraso das primeiras chuvas ou ocorrência de veranico (LIMA et al., 2008) também prejudicam o re- crutamento das plantas. Em razão disso, a produção de mudas em condições controladas pode facilitar a inserção de novos indivíduos nos fragmentos, auxiliando na manutenção da espécie.

Assim, neste estudo procurou-se analisar a germinação das sementes de Anadenanthera colubrina, oriundas de fragmentos antropizados do Cerrado, pressupondo-se que por meio desta análise seja possível avaliar a eficiência da espécie para sobreviver. Deste modo, o processo de germinação das sementes e a emergência de plântulas foram analisados com base na produção de cinco indivíduos ocorrentes no Vale do Rio Araguari, o que também permitiu avaliar a variabilidade entre eles.

\section{MATERIAIS E MÉTODOS}

\section{Área de coleta}

A coleta dos frutos foi realizada no Vale do Rio Araguari, MG, no final de agosto de 2005, em trechos situados entre as coordenadas 795360 - 7918682 S e 810533 - 7909694 N UTM (Figura 1). A cobertura vegetal do Vale encontra-se fragmentada, apresentando remanescentes da fitofisionomia do Bioma Cerrado (FERRETE et al., 2005).

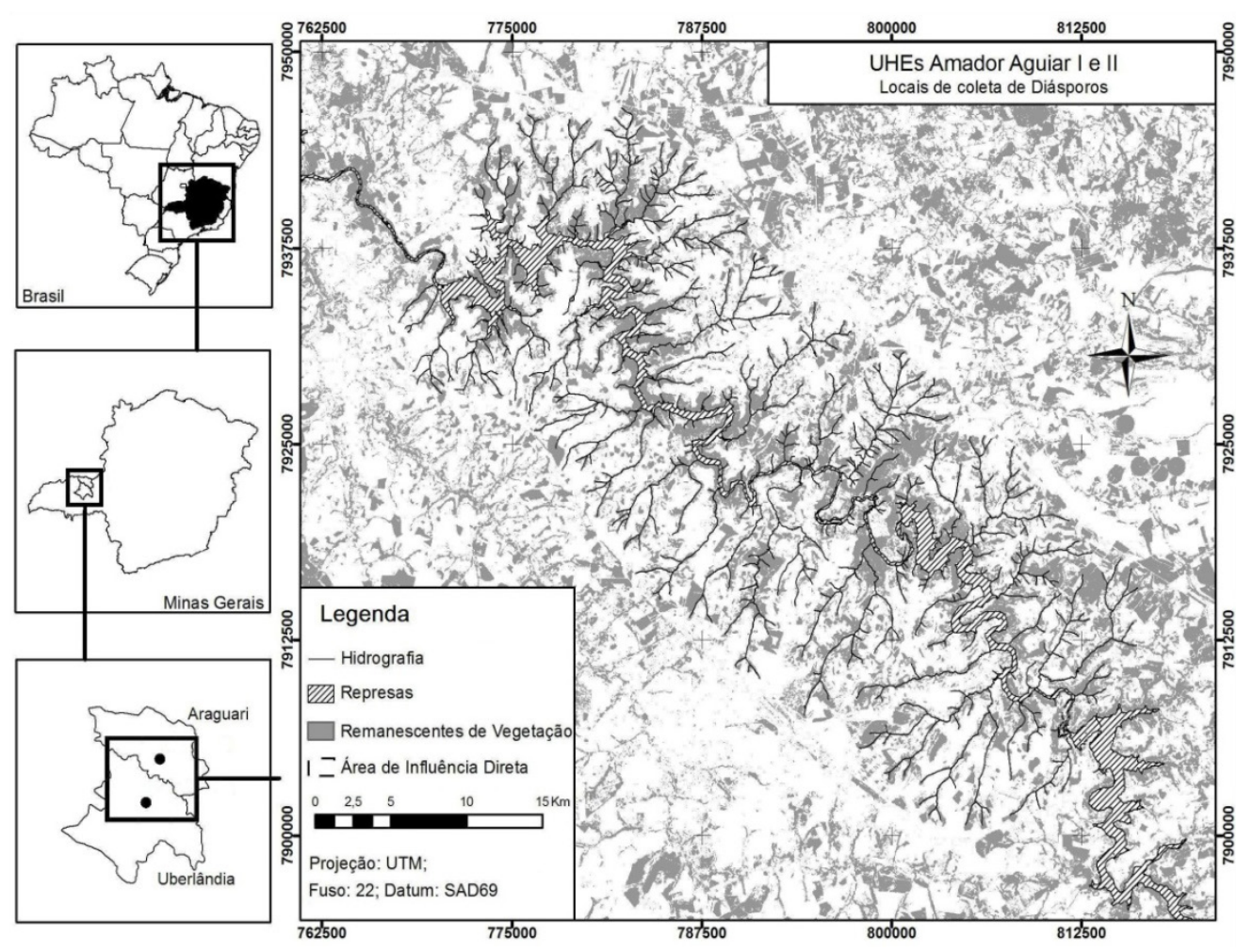

FIGURA 1: Localização geográfica do Vale do Rio Araguari, MG

FIGURE 1: Geographical location of Araguari River Valley, MG state. 
A região é caracterizada pelo tipo climático Aw, segundo o sistema de classificação de Köppen (1948), considerado tropical úmido com inverno seco (abril a setembro) e verão chuvoso (outubro a março). De acordo com registros do sistema meteorológico de Minas Gerais, durante o ano de coleta em 2005, o Vale do Rio Araguari apresentou índice pluviométrico de $1300 \mathrm{~mm}$; temperaturas médias, mínimas e máximas de 25,19 e $31^{\circ} \mathrm{C}$, respectivamente (SIMGE, 2010).

\section{Coleta de sementes}

As sementes analisadas foram coletadas a partir de cinco indivíduos de Anadenanthera colubrina, com distância mínima entre eles de aproximadamente $3 \mathrm{~km}$ (Tabela 1), alguns inseridos na vegetação remanescente e outros isolados, em áreas de pastagens. Esses foram os únicos indivíduos férteis localizados no período de 2004 a 2007, em excursões semanais.

Após a coleta dos frutos, as sementes foram beneficiadas manualmente e selecionadas as que se apresentavam em melhores condições morfológicas, separando-se aquelas com danos naturais ou ocorridos durante a coleta ou beneficiamento. Em

TABELA 1: Data de coleta, posição geográfica e caracterização dos indivíduos dos quais as sementes de Anadenanthera colubrina (Vell.) Brenan var. cebil (Griseb.) Altschut foram coletadas. Coleta feita no Vale do Rio Araguari, MG, em 2005.

TABLE 1: Collection date, geographic position and characteristics of the individuals from which the seeds of Anadenanthera colubrina (Vell.) Brenan var. cebil (Griseb.) Altschut were collected. Collection made in Araguari River Valley, MG state, 2005.

\begin{tabular}{ccccc}
\hline Indivíduo & \multirow{2}{*}{ Coleta } & UTM & $\begin{array}{c}\text { ALT } \\
(\mathrm{m})\end{array}$ & $\begin{array}{c}\text { CAP } \\
(\mathrm{m})\end{array}$ \\
\hline 1 & $23 / 08 / 2005$ & $0805732-7911404$ & 18 & 1,32 \\
2 & $23 / 08 / 2005$ & $0810533-7909694$ & 18 & 2,37 \\
3 & $25 / 08 / 2005$ & $0802398-7912324$ & 20 & 1,88 \\
4 & $25 / 08 / 2005$ & $0803138-7916838$ & 22 & 1,82 \\
5 & $30 / 08 / 2005$ & $0795360-7918682$ & 19 & 2,57 \\
\hline
\end{tabular}

Em que: UTM = Universal Transverse Mercator; ALT $=$ altura; $\mathrm{CAP}=$ circunferência à altura do peito. seguida, determinou-se o teor de água e os processos de germinação e emergência foram analisados.

\section{Teor de água}

O teor de água das sementes foi avaliado a $105^{\circ} \mathrm{C}$, até massa constante. Oito repetições com 17 sementes para cada um dos indivíduos, com massa média por repetição de $5,8 \mathrm{~g}$, foram utilizadas. $\mathrm{O}$ cálculo do teor de água foi feito com base na massa seca (teor de água $=(m m f-m m s / m m s) 100$, onde $m m f$ : massa da matéria fresca e $m m s$ : massa da matéria seca).

\section{Germinação}

O experimento foi instalado em delineamento inteiramente casualizado, utilizando-se sementes dos cinco indivíduos e quatro repetições de 25 sementes. As sementes foram distribuídas em caixas tipo gerbox, sobre $128 \mathrm{~cm}^{3}$ de vermiculita expandida a $0,1 \mathrm{~m}^{3}$, umedecida com $65 \mathrm{~mL}$ de água destilada e mantidas em câmara de germinação (Seedburo Equipment Company, modelo MPG2000) sob luz branca fluorescente contínua, irradiância média de $12,6 \pm 6,3 \mu \mathrm{mol} \mathrm{m}^{-2} \mathrm{~s}^{-1}$; entre $25,8 \pm$ 0,7 e $26,9 \pm 1,2^{\circ} \mathrm{C}$.

\section{Emergência}

O experimento foi instalado em delineamento de blocos casualizados, com sementes dos cinco indivíduos e quatro parcelas contendo 32 sementes. A semeadura foi feita a $1 \mathrm{~cm}$ de profundidade, em bandejas de poliestireno multicelulares preenchidas com vermiculita expandida e substrato comercial (1:1), mantendo-se irrigações diárias. O experimento foi instalado em estufa coberta com sombrite, com $50 \%$ de redução de luz, sob irradiância média de $136,4 \pm 14,7 \mu \mathrm{mol} \mathrm{m}^{-2} \mathrm{~s}^{-1}$, entre $18,7 \pm 1,4$ e 29,9 $\pm 1,2{ }^{\circ} \mathrm{C}$.

\section{Critério de avaliação e medidas calculadas}

Os experimentos foram monitorados diariamente, avaliando-se a protrusão do embrião para a germinação e a emissão de qualquer parte da plântula acima do substrato, para a emergência. A partir da coleta dos dados, foram calculadas as medidas de germinação e emergência, incluindo-se germinabilidade, porcentagem de emergência, tempo médio, tempo inicial e final, velocidade média, coeficiente 
de variação do tempo, incerteza e sincronia de ambos os processos (RANAL e SANTANA, 2006).

\section{Análise estatística}

Os dados foram submetidos ao teste de Shapiro-Wilk para a normalidade dos resíduos da ANOVA e de Levene para a homogeneidade entre as variâncias. Atendidas a essas duas pressuposições da estatística paramétrica, aplicou-se a análise da variância (ANOVA), seguida do teste de Tukey para comparações entre as médias $(\alpha=0,05)$. Para o tempo final $\left(t_{f}\right)$ de germinação, cujos resíduos não apresentaram normalidade, utilizou-se o teste de Kruskal-Wallis seguido de Dunn para comparações múltiplas $(\alpha=0,05)$.

\section{RESULTADOS E DISCUSSÃO}

Sementes de Anadenanthera colubrina apresentaram baixo teor de água, variando de 8,0 a $10,7 \%$ (Tabela 2). Esta parece ser uma característica da espécie, sendo registrada também para sementes coletadas nos municípios de Três Barras, PR (GARCIA et al., 2004), na Bacia do Alto e Médio Rio Grande do município de Lavras, MG (CARVALHO et al., 2006), no Vale do Rio Paranã, nordeste de Goiás (LIMA et al., 2008) e nos municípios do semiárido de Tanquinho e Cruz das Almas, BA (RODRIGUES et al., 2006). O baixo teor de água das sementes anidrobióticas de $A$. colubrina permite sua conservação ex situ por 90 dias, sob baixa temperatura e umidade (CARVALHO et al., 2006). Isto as torna capazes de se manterem viá-

TABELA 2: Teor de água (média \pm desvio padrão) das sementes de Anadenanthera colubrina (Vell.) Brenan var. cebil (Griseb.) Altschut, coletadas no Vale do Rio Araguari, MG.

TABLE 2: Water content (mean \pm standard deviation) of Anadenanthera colubrina (Vell.) Brenan var. cebil (Griseb.) Altschut seeds collected in Araguari River Valley, MG state.

\begin{tabular}{cc}
\hline Indivíduo & $105^{\circ} \mathrm{C}$ \\
\hline 1 & $8,8 \pm 0,8$ \\
2 & $8,1 \pm 0,3$ \\
3 & $9,5 \pm 0,4$ \\
4 & $10,7 \pm 3,7$ \\
5 & $8,0 \pm 0,3$ \\
\hline
\end{tabular}

veis no ambiente, formando banco de sementes. Sementes de A. peregrina (L.) Speg. encontradas no banco de sementes da serapilheira de um fragmento da floresta estacional semidecidual em Viçosa, MG (SOUZA et al., 2006) também são anidrobióticas e germinam após 22 meses de armazenamento sob baixa temperatura e umidade (BORGES et al., 2009).

A germinabilidade e a porcentagem de emergência das plântulas de Anadenanthera colubrina foram altas, exceto para as sementes oriundas do indivíduo 2 , o que gerou a variabilidade detectada na análise estatística (Tabelas 3 e 4). Morfologicamente as sementes desse indivíduo não apresentaram características visuais que poderiam justificar o seu menor desempenho nos processos analisados. Além disso, a germinação das sementes desse indivíduo foi de $68 \%$ (Tabela 3 ) e isso indica a presença de embrião fisiologicamente funcional em grande parte das unidades estudadas; porém, com baixo vigor, o que acarretou a baixa porcentagem de emergência (Tabela 4). Em condições de campo, sob as adversidades climáticas e ecológicas, as sementes desse indivíduo certamente seriam mais afetadas do que as demais. Complicações desse tipo podem afetar a regeneração natural na floresta, por limitar o recrutamento nas fases iniciais do ciclo de vida das plantas (ALVES e METZGER, 2006). Além disso, para a produção de mudas com finalidade de revegetação do ambiente, a limitação de sementes oriundas de apenas um indivíduo com baixo desempenho pode comprometer o processo, em função da inclusão no sistema de plantas menos vigorosas e com menor aptidão para superar as adversidades naturais. Por outro lado, a produção de sementes, mesmo que menos vigorosas, pode estar retratando o esforço reprodutivo da planta-mãe e, quando essas são somadas às demais sementes, produzidas por outros indivíduos da população, a variabilidade local é aumentada, fato essencial para a conservação das espécies no ambiente, como apontam Kageyama et al. (2003), dentre outros autores.

As sementes de Anadenanthera colubrina coletadas no Vale do Rio Araguari apresentaram rapidez para a germinação e emergência (ver valores de $t_{0}, t_{f} ; \bar{t}$ e $\bar{v}$; Tabelas 3 e 4). Este comportamento também foi registrado para sementes desta espécie, coletadas no município de Três Barras, PR, podendo-se inferir a partir das frequências relativas de germinação que o tempo médio está dentro do intervalo de 5 a 7 dias (GARCIA et al., 2004). A rapidez para a germinação e emergência mostra a es- 
TABELA 3: Medidas de germinação (média \pm desvio padrão) de sementes de Anadenanthera colubrina (Vell.) Brenan var. cebil (Griseb.) Altschut coletadas no Vale do Rio Araguari, MG, a partir de cinco indivíduos.

TABLE 3: Germination measurements (mean \pm standard deviation) of Anadenanthera colubrina (Vell.) Brenan var. cebil (Griseb.) Altschut seeds collected in Araguari River Valley, MG state, from five individuals.

\begin{tabular}{cccccc}
\hline Indivíduo & ${ }^{3} G(\%)$ & $t_{0}($ dia $)$ & $t_{f}($ dia $)$ & $\bar{t}($ dia $)$ & $\bar{v}\left(\right.$ dia $\left.^{-1}\right)$ \\
\hline 1 & $94,0 \pm 5,2 \mathrm{a}$ & $2,0 \pm 0,8 \mathrm{a}$ & $10,5 \pm 5,2 \mathrm{a}$ & $4,0 \pm 0,4 \mathrm{~b}$ & $0,2464 \pm 0,0249 \mathrm{~b}$ \\
2 & $68,0 \pm 21,7 \mathrm{~b}$ & $2,2 \pm 0,5 \mathrm{a}$ & $12,8 \pm 9,8 \mathrm{a}$ & $3,9 \pm 0,4 \mathrm{ab}$ & $0,2628 \pm 0,0316 \mathrm{ab}$ \\
3 & $86,0 \pm 5,2 \mathrm{ab}$ & $1,5 \pm 1,0 \mathrm{a}$ & $7,0 \pm 0,0 \mathrm{a}$ & $4,2 \pm 0,1 \mathrm{~b}$ & $0,2363 \pm 0,0072 \mathrm{~b}$ \\
4 & $85,0 \pm 6,8 \mathrm{ab}$ & $1,0 \pm 0,0 \mathrm{a}$ & $8,8 \pm 2,0 \mathrm{a}$ & $3,6 \pm 0,2 \mathrm{ab}$ & $0,2816 \pm 0,0136 \mathrm{ab}$ \\
5 & $87,0 \pm 2,0 \mathrm{ab}$ & $1,2 \pm 0,5 \mathrm{a}$ & $5,0 \pm 1,2 \mathrm{a}$ & $3,2 \pm 0,4 \mathrm{a}$ & $0,3153 \pm 0,0378 \mathrm{a}$ \\
\hline$W(P)$ & $\mathbf{0 , 9 4 3 9}(0,2959)$ & $\mathbf{0 , 8 7 0 8}(0,0114)$ & $\mathbf{0 , 9 4 5 7}(0,3190)$ & $\mathbf{0 , 9 6 1 9}(0,5848)$ & $\mathbf{0 , 9 9 0 1}(0,9951)$ \\
${ }^{l} F(P)$ & $\mathbf{2 , 3 8 0}(0,0981)$ & $\mathbf{2 , 0 7 1}(0,1355)$ & $28,78(0,0001)$ & $\mathbf{3 , 5 3 4}(0,0319)$ & $\mathbf{2 , 1 9 8}(0,1186)$ \\
${ }^{2} F(P)$ & $\mathbf{3 , 2 2 7}(0,0425)$ & $2,48(0,0885)$ & & $\mathbf{5 , 8 6}(0,0048)$ & $\mathbf{5 , 9 7 3}(0,0044)$ \\
$H(P)$ & & & $5,481(0,2414)$ & & \\
\hline
\end{tabular}

Em que: $G=$ germinabilidade; $t_{0}=$ tempo para a primeira germinação; $t_{f}=$ tempo para a última germinação; $\bar{t}=$ tempo médio de germinação; $\bar{v}=$ velocidade média de germinação; médias seguidas por letras iguais na coluna não diferem entre si pelos testes de Tukey ou Dunn a 0,05 de significância; $W=$ estatística do teste de Shapiro-Wilk; valores em negrito indicam que os resíduos seguem distribuição normal $(P>0,01) ;{ }^{1} F=$ estatística do teste de Levene; valores em negrito indicam homogeneidade entre as variâncias $(P>0,01) ;{ }^{2} F=$ estatística do teste de Snedecor; valores em negrito indicam diferença significativa entre os indivíduos (ANOVA; $P<0,05$ ); $H=$ estatística do teste de Kruskal-Wallis; valores em negrito indicam diferença significativa entre os indivíduos $(P<0,05) ; P=$ probabilidade; ${ }^{3}$ comparações múltiplas efetuadas com dados transformados em arcoseno(raiz(x/100).

TABELA 4: Medidas de emergência de plântulas (média \pm desvio padrão) de Anadenanthera colubrina (Vell.) Brenan var. cebil (Griseb.) Altschut oriundas de sementes coletadas no Vale do Rio Araguari, MG, a partir de cinco indivíduos.

TABLE 4: Seedling emergence measurements (mean \pm standard deviation) of Anadenanthera colubrina (Vell.) Brenan var. cebil (Griseb.) Altschut from seeds collected in Araguari River Valley, MG state from five individuals.

\begin{tabular}{cccccc}
\hline Indivíduo & $E(\%)$ & $t_{0}$ (dia) & $t_{f}(\mathrm{dia})$ & $\bar{t}(\mathrm{dia})$ & $\bar{v}\left(\mathrm{dia}^{-1}\right)$ \\
\hline 1 & $90,6 \pm 5,1 \mathrm{a}$ & $4,5 \pm 1,0 \mathrm{a}$ & $15,5 \pm 6,2 \mathrm{ab}$ & $7,5 \pm 0,3 \mathrm{~b}$ & $0,1331 \pm 0,0057 \mathrm{~b}$ \\
2 & $38,2 \pm 5,9 \mathrm{~b}$ & $4,2 \pm 1,0 \mathrm{a}$ & $10,5 \pm 1,8 \mathrm{a}$ & $6,1 \pm 0,4 \mathrm{a}$ & $0,1643 \pm 0,0098 \mathrm{a}$ \\
3 & $83,6 \pm 3,9 \mathrm{a}$ & $4,5 \pm 1,2 \mathrm{a}$ & $13,2 \pm 1,9 \mathrm{ab}$ & $7,2 \pm 0,5 \mathrm{~b}$ & $0,1386 \pm 0,0095 \mathrm{ab}$ \\
4 & $78,1 \pm 5,7 \mathrm{a}$ & $3,2 \pm 0,5 \mathrm{a}$ & $17,0 \pm 5,0 \mathrm{ab}$ & $6,4 \pm 0,8 \mathrm{ab}$ & $0,1563 \pm 0,0179 \mathrm{ab}$ \\
5 & $79,7 \pm 7,4 \mathrm{a}$ & $3,0 \pm 0,0 \mathrm{a}$ & $18,8 \pm 4,2 \mathrm{~b}$ & $6,6 \pm 1,0 \mathrm{ab}$ & $0,1535 \pm 0,0237 \mathrm{ab}$ \\
\hline$W(P)$ & $\mathbf{0 , 9 5 4}(0,4523)$ & $\mathbf{0 , 9 3 1}(0,1708)$ & $\mathbf{0 , 9 5 0}(0,3727)$ & $\mathbf{0 , 9 7 6}(0,8518)$ & $\mathbf{0 , 9 7 2}(0,7857)$ \\
${ }^{I} F(P)$ & $\mathbf{1 , 0 9 5}(0,3946)$ & $\mathbf{3 , 7 6 7}(0,0259)$ & $\mathbf{1 , 4 4 7}(0,2670)$ & $\mathbf{1 , 4 1 2}(0,2779)$ & $\mathbf{1 , 8 1 3}(0,1789)$ \\
${ }^{2} F(P)$ & $\mathbf{4 4 , 1 8 5}(0,0001)$ & $2,706(0,0705)$ & $\mathbf{4 , 4 7 2}(0,0192)$ & $\mathbf{5 , 7 2 4}(0,0082)$ & $\mathbf{4 , 7 9 0}(0,0153)$ \\
\hline
\end{tabular}

Em que: $E=$ porcentagem de emergência; $t_{0}=$ tempo para a primeira emergência; $t_{f}=$ tempo para a última emergência; $\bar{t}=$ tempo médio de emergência; $\bar{v}=$ velocidade média de emergência; médias seguidas por letras iguais na coluna não diferem entre si pelos testes de Tukey ou Dunn a 0,05 de significância; $W=$ estatística do teste de Shapiro-Wilk; valores em negrito indicam que os resíduos seguem distribuição normal $(P>0,01) ;{ }^{1} F=$ estatística do teste de Levene; valores em negrito indicam homogeneidade entre as variâncias $(P>0,01) ;{ }^{2} F=$ estatística do teste de Snedecor; valores em negrito indicam diferença significativa entre os indivíduos (ANOVA; $P<0,05$ ); $P$ = probabilidade. 
tratégia da espécie para sobreviver no ambiente pelo favorecimento da formação do banco de plântulas com as primeiras chuvas. Cerca de 100 sementes germinadas por $\mathrm{m}^{2}$ sob a planta-mãe dessa espécie foram observadas na Caatinga de Sertânia, PE (BARBOSA, 2003). Segundo esta autora, a rapidez de germinação das sementes desta espécie pode estar relacionada com a morfologia das sementes que apresentam tegumento delicado e pleurograma ou linha fissural na testa.

Esta característica morfológica de sementes é utilizada para fins taxonômicos e evolutivos e sua função permanece desconhecida. No levantamento bibliográfico apresentado por De-Paula e Oliveira (2008), os autores resgatam três trabalhos importantes relacionados com possíveis pontos de comunicação entre a semente e o ambiente, que são o hilo como válvula higrométrica de mínima, detectado como tal em um experimento realizado por Hyde (1954) e dois trabalhos de Gunn $(1981,1991)$ em que o autor associa pleurograma com a desidratação das sementes de Mimosoideae e Caesalpinioideae; porém, sem dados experimentais comprobatórios. Como não há fissura verdadeira nessa linha lúcida denominada pleurograma, somente dados que possam mensurar especificamente a velocidade de passagem de água por ela poderiam esclarecer a questão. Para Anadenanthera colubrina, com se- mentes mais macias do que as de outras espécies convencionalmente consideradas com testa dura e impermeável, a entrada de água por todo o tegumento parece ser a explicação mais apropriada. A permeabilidade do tegumento, detectada pela alta velocidade de germinação, poderia explicar o rápido estabelecimento da espécie em ambientes perturbados, conforme observado por Lopes et al. (2002).

A germinação e a emergência ocorreram com heterogeneidade para as sementes de todos os indivíduos analisados, com valores do coeficiente de variação do tempo acima de $28,5 \%$ (Tabelas 5 e 6). Isto pode ser melhor observado analisando-se as frequências relativas de germinação e emergência (Figuras 2 e 3). Entre 1 e 5 dias após a semeadura, foram registrados $40 \%$ de germinação ou emergência, sendo o restante espalhado ao longo de 25 dias. A falta de homogeneidade registrada para os processos de germinação e emergência de Anadenanthera colubrina parece ser um comportamento comum entre as espécies nativas e isso foi detectado também para núculas de Anacardium humile A. St.-Hil. (CARVALHO et al., 2005); para a germinação de diásporos de Myracrodruon urundeuva Allemão (DORNELES et al., 2005), embora maior homogeneidade tenha sido registrada para a emergência das plântulas desta espécie (valores de $C V_{t}$ entre 11,20 e $20,71 \%$; BERGER et al., 2007); para se-

TABELA 5: Medidas de germinação (média \pm desvio padrão) de sementes de Anadenanthera colubrina (Vell.) Brenan var. cebil (Griseb.) Altschut coletadas no Vale do Rio Araguari, MG, a partir de cinco indivíduos.

TABLE 5: Germination measurements (mean \pm standard deviation) of Anadenanthera colubrina (Vell.) Brenan var. cebil (Griseb.) Altschut seeds collected in Araguari River Valley, MG state, from five individuals.

\begin{tabular}{cccc}
\hline Indivíduo & ${ }^{3} C V_{\mathrm{t}}(\%)$ & $I$ (bit) & $Z$ \\
\hline 1 & $50,0 \pm 23,0 \mathrm{a}$ & $1,6 \pm 0,2 \mathrm{a}$ & $0,3812 \pm 0,0434 \mathrm{a}$ \\
2 & $58,8 \pm 44,1 \mathrm{a}$ & $1,5 \pm 0,4 \mathrm{a}$ & $0,3341 \pm 0,0900 \mathrm{ab}$ \\
3 & $29,5 \pm 2,2 \mathrm{a}$ & $2,0 \pm 0,2 \mathrm{ab}$ & $0,2810 \pm 0,0744 \mathrm{ab}$ \\
4 & $49,0 \pm 12,1 \mathrm{a}$ & $2,4 \pm 0,2 \mathrm{~b}$ & $0,2170 \pm 0,0566 \mathrm{~b}$ \\
5 & $34,2 \pm 6,4 \mathrm{a}$ & $1,9 \pm 0,2 \mathrm{ab}$ & $0,2872 \pm 0,0612 \mathrm{ab}$ \\
\hline$W(P)$ & $\mathbf{0 , 9 6 4 2}(0,63)$ & $\mathbf{0 , 9 5 7}(0,4927)$ & $\mathbf{0 , 9 7 7 6}(0,8834)$ \\
${ }^{1} F(P)$ & $\mathbf{0 , 5 9}(0,6753)$ & $\mathbf{0 , 8 3 9}(0,5214)$ & $\mathbf{0 , 3 8 5}(0,8162)$ \\
${ }^{2} F(P)$ & $3,929(0,0224)$ & $\mathbf{5 , 2 3 2}(0,0077)$ & $\mathbf{3 , 8 6 7}(0,0236)$ \\
\hline
\end{tabular}

Em que: $C V_{\mathrm{t}}=$ coeficiente de variação do tempo; $I=$ incerteza; $Z=$ sincronia; médias seguidas por letras iguais na coluna não diferem entre si pelos testes de Tukey ou Dunn a 0,05 de significância; $W=$ estatística do teste de ShapiroWilk; valores em negrito indicam que os resíduos seguem distribuição normal $(P>0,01) ;{ }^{1} F=$ estatística do teste de Levene; valores em negrito indicam homogeneidade entre as variâncias $(P>0,01) ;{ }^{2} F=$ estatística do teste de Snedecor; valores em negrito indicam diferença significativa entre os indivíduos (ANOVA; $P<0,05$ ); valores em negrito indicam diferença significativa entre os indivíduos $(P<0,05) ; P=$ probabilidade, ${ }^{3}$ comparações múltiplas efetuadas com dados transformados em arcoseno(raiz(x/100). 
TABELA 6: Medidas de emergência de plântulas (média \pm desvio padrão) de Anadenanthera colubrina (Vell.) Brenan var. cebil (Griseb.) Altschut oriundas de sementes coletadas no Vale do Rio Araguari, MG, a partir de cinco indivíduos.

TABLE 6: Seedling emergence measurements (mean \pm standard deviation) of Anadenanthera colubrina (Vell.) Brenan var. cebil (Griseb.) Altschut from seeds collected in Araguari River Valley, MG state, from five individuals.

\begin{tabular}{cccc}
\hline Indivíduo & $C V_{\mathrm{t}}(\%)$ & $I(\mathrm{bit})$ & $Z$ \\
\hline 1 & $30,0 \pm 11,0 \mathrm{a}$ & $2,6 \pm 0,2 \mathrm{a}$ & $0,1683 \pm 0,0170 \mathrm{a}$ \\
2 & $28,5 \pm 10,6 \mathrm{a}$ & $2,0 \pm 0,4 \mathrm{a}$ & $0,2526 \pm 0,0895 \mathrm{a}$ \\
3 & $30,0 \pm 5,4 \mathrm{a}$ & $2,4 \pm 0,4 \mathrm{a}$ & $0,2104 \pm 0,0690 \mathrm{a}$ \\
4 & $48,1 \pm 14,0 \mathrm{ab}$ & $2,6 \pm 0,5 \mathrm{a}$ & $0,1792 \pm 0,0914 \mathrm{a}$ \\
5 & $54,9 \pm 16,4 \mathrm{~b}$ & $2,6 \pm 0,2 \mathrm{a}$ & $0,1758 \pm 0,0330 \mathrm{a}$ \\
\hline$W(P)$ & $\mathbf{0 , 9 5 5}(0,4643)$ & $\mathbf{0 , 9 5 4}(0,4371)$ & $\mathbf{0 , 9 6 3 4}(0,6135)$ \\
${ }^{l} F(P)$ & $\mathbf{1 , 0 2 4}(0,4264)$ & $\mathbf{1 , 5 0 1}(0,2518)$ & $\mathbf{2 , 2 0 0}(0,1183)$ \\
${ }^{2} F(P)$ & $\mathbf{6 , 8 2 9}(0,0042)$ & $2,078(0,1470)$ & $1,194(0,3645)$ \\
\hline
\end{tabular}

Em que: $C V_{\mathrm{t}}=$ coeficiente de variação do tempo; $I=$ incerteza; $Z$ = sincronia; médias seguidas por letras iguais na coluna não diferem entre si pelos testes de Tukey ou Dunn a 0,05 de significância; $W=$ estatística do teste de Shapiro-Wilk; valores em negrito indicam que os resíduos seguem distribuição normal $(P>0,01) ;{ }^{1} F=$ estatística do teste de Levene; valores em negrito indicam homogeneidade entre as variâncias $(P>0,01) ;{ }^{2} F=$ estatística do teste de Snedecor; valores em negrito indicam diferença significativa entre os indivíduos (ANOVA; $P<0,05$ ); $P=$ probabilidade.

mentes de Luehea divaricata Mart. (BORGES et al., 2007), de Inga laurina Willd. (MENDESRODRIGUES et al., 2007), de Miconia ferruginata DC. (MENDES-RODRIGUES et al., 2010) e para a maioria das 19 espécies florestais estudadas por Dorneles (2010). Esta heterogeneidade pode estar refletindo as oscilações climáticas e ecológicas que ocorreram durante os eventos de polinização, formação e maturação dos frutos e sementes, conforme apontou Garwood (1983). É interessante observar que, para uma espécie com germinação rápida como I. laurina, com tempo médio de 1 dia, a heterogeneidade tenha valores entre 24 e $38 \%$ (MENDES-RODRIGUES et al., 2007), enquanto para Copaifera langsdorffii Desf. que apresenta sementes com dormência múltipla, os coeficientes de variação do tempo estejam entre 12 e $23 \%$ (PEREIRA et al., 2009). Como 2005 foi um ano de baixa produção para $C$. langsdorffii na região do Triângulo Mineiro, provavelmente as sementes que chegaram até o final da maturação tenham sido mais similares entre si do que seriam em um ano de alta produção, apresentando-se então homogêneas. Além dessas pressões seletivas do ambiente, a heterogeneidade das sementes e o espalhamento da germinação no tempo garantem que, pelo menos parte da safra produzida naquele ano, possa dar origem a plântulas que consigam se estabelecer no local.

Os menores valores de incerteza e os maiores de sincronia para a germinação, comparados com os valores da emergência, mostram que no primeiro processo as sementes tiveram menor assincronia do que no segundo, como consequência da maior estabilidade das condições térmicas e de umidade do ambiente de germinação (Tabelas 5 e 6). Além disso, na germinação, apenas a protrusão da radícula é avaliada e isso não depende da alta qualidade da semente; porém, para o desenvolvimento da plântula, este requisito é necessário para vencer alguns milímetros de barreira que o solo representa. Entretanto, a assincronia registrada em ambos os processos reflete o efeito da desuniformidade dessas unidades. Alterações na temperatura e umidade, posição do fruto na planta-mãe e posição da semente no fruto são alguns dos fatores que podem gerar desuniformidade na germinação (BASKIN e BASKIN, 1998). Esse efeito é mais evidente nas espécies com períodos longos entre a floração e a formação das sementes (CASTRO et al., 2004). Talvez o intervalo de tempo de aproximadamente 10 meses para os eventos fenológicos de Anadenanthera colubrina, com início da floração em novembro, finalizando com a dispersão das sementes em setembro do ano seguinte (CARVALHO, 2003), tenha propiciado a desuniformidade morfofisiológica nas sementes e gerado a assincronia da germinação.

O presente estudo possibilitou verificar a eficiência dos processos de germinação e emergência das plântulas oriundas de sementes coletadas a partir de alguns indivíduos de Anadenanthera colubrina 

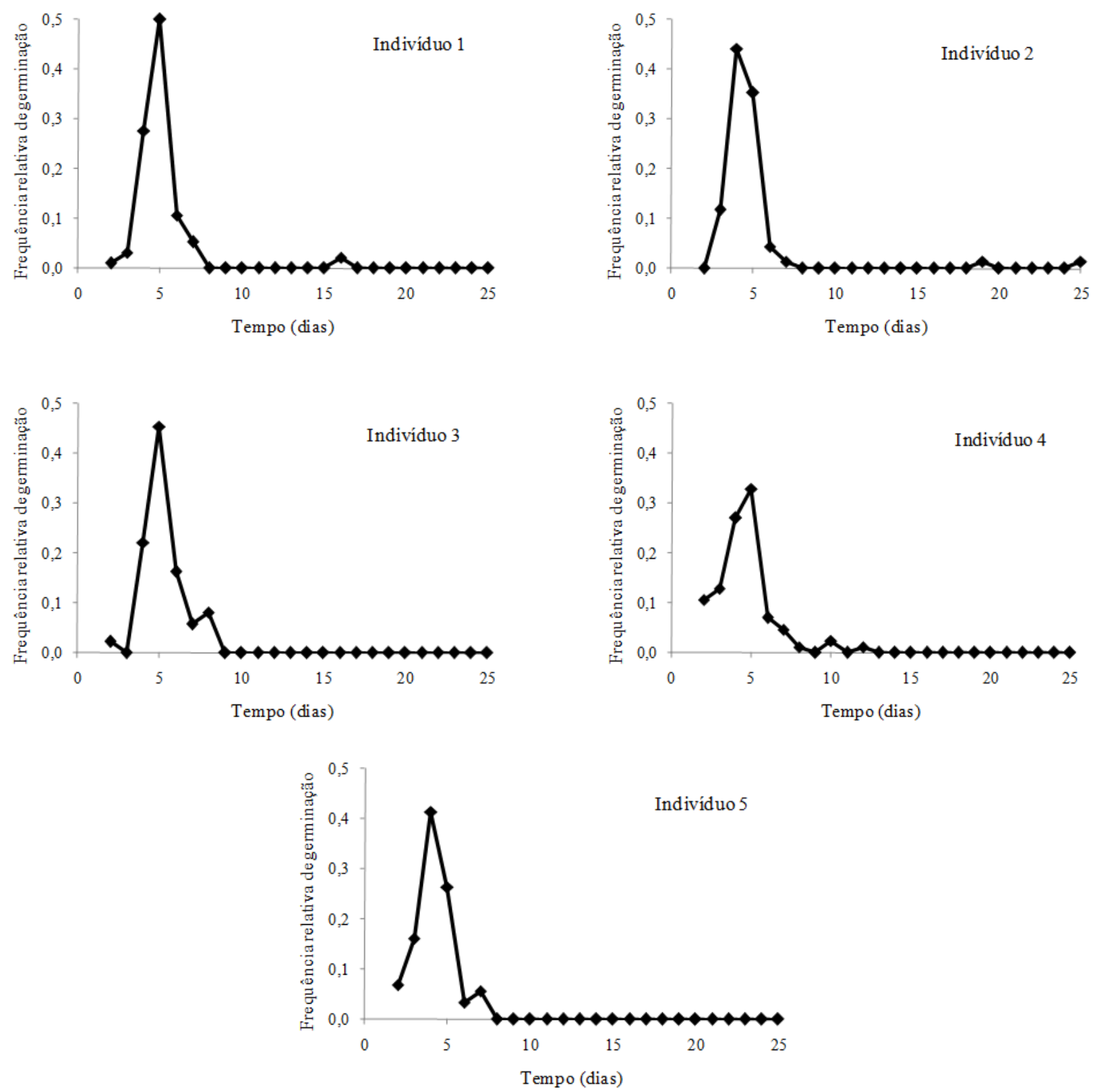

FIGURA 2: Frequência relativa de germinação das sementes oriundas de cinco indivíduos de Anadenanthera colubrina (Vell.) Brenan var. cebil (Griseb.) Altschut estabelecidos no Vale do Rio Araguari, MG.

FIGURE 2: Relative frequency of seed germination of five Anadenanthera colubrina (Vell.) Brenan var. cebil (Griseb.) Altschut individuals established in Araguari River Valley, MG state.

estabelecidos nos fragmentos florestais do Cerrado. Os altos valores encontrados para a germinabilidade e porcentagem de emergência indicam que as alterações decorrentes da fragmentação não afetaram a formação das sementes no ano da coleta. Essa habilidade parece ser intrínseca dessa espécie, sendo que também foi observado acima de $75 \%$ de germinação para as sementes oriundas de fragmentos florestais dos municípios de Três Barras e Colombo, PR, e do Vale do Rio Paranã, GO (GARCIA et al., 2004; REGO et al., 2007; LIMA et al., 2008). Além disso, a rapidez dos processos avaliados retrata a estratégia da espécie para sobreviver (BARBOSA, 2003; CARVALHO, 2003). Após a dispersão e com a entrada de umidade no ambiente, as sementes podem germinar rapidamente, garantindo a sobrevivência da espécie. Esse comportamento mostra a característica funcional de $A$. colubrina para se manter no 
ambiente. Atributos biológicos comuns como preferência por certos hábitats, fenologia das folhas, tipos de dispersão e estratégias reprodutivas, dentre outros (DECKERS et al., 2004), são respostas das espécies vegetais a determinados fatores ambientais para se estabelecerem, o que permite a identificação de grupos funcionais (LAVOREL et al., 1998).

A rapidez e o alto potencial de germinação das sementes desta espécie também podem viabilizar programas de recuperação ou revegetação, com a produção de grande quantidade de plantas jovens em menor tempo. Após o plantio, o estabelecimento dos indivíduos pode ocorrer, pois Anadenathera colubrina está classificada no grupo sucessional das secundárias iniciais e isto favorece o seu crescimento em pequenas áreas abertas (PAULA et al., 2004). Além disso, a presença dessa espécie em fragmentos estudados por diversos pesquisadores aponta a sua capacidade para se estabelecer em ambientes antropizados (PAULA et al., 2004).
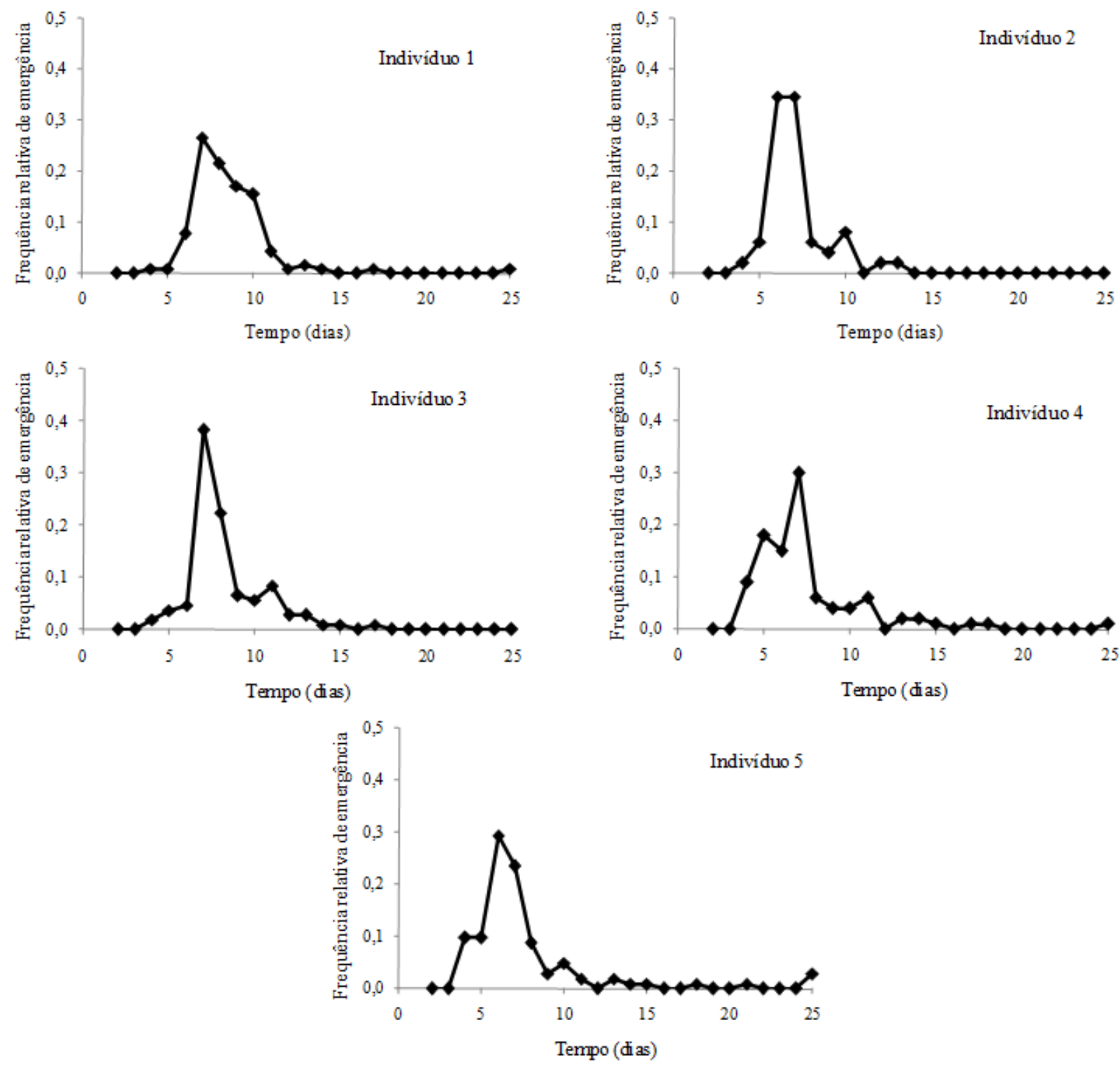

FIGURA 3: Frequência relativa de emergência das plântulas oriundas de cinco indivíduos de Anadenanthera colubrina (Vell.) Brenan var. cebil (Griseb.) Altschut. estabelecidos no Vale do Rio Araguari, MG.

FIGURE 3: Relative frequency of seedling emergence of five Anadenanthera colubrina (Vell.) Brenan var. cebil (Griseb.) Altschut individuals established in Araguari River Valley, MG state. 


\section{CONCLUSÕES}

A eficiência da germinação e emergência das plântulas de Anadenanthera colubrina mostrou a aptidão da espécie para sobreviver, embora as sementes de um dos indivíduos tenham apresentado baixa qualidade. Do ponto de vista ecológico, as sementes desse indivíduo atípico, para o conjunto estudado, que se desenvolveram até a fase de plântula, podem ter retratado o seu esforço reprodutivo para superar as adversidades do ambiente.

\section{AGRADECIMENTOS}

À equipe de coleta de sementes coordenada por Sérgio Farias Lopes, aos estagiários do Laboratório de Ecofisiologia Vegetal (2004-2007) da Universidade Federal de Uberlândia, à CAPES pela bolsa concedida à primeira autora e à FAPEMIG pelo auxílio financeiro.

\section{REFERÊNCIAS BIBLIOGRÁFICAS}

ALHO, C. J. R. Desafios para a conservação do Cerrado, em face das atuais tendências de uso e ocupação. In: SCARLOT, A.; SOUZA-SILVA, J. C.; FELFILI, J. M. (orgs.). Cerrado: biologia, biodiversidade e conservação. Brasília: Ed. do Ministério do Meio Ambiente. 2005, p. 369-381.

ALVES, L. F.; METZGER, J. P. A regeneração florestal em áreas de floresta secundária na Reserva Florestal do Morro Grande, Cotia, SP. Biota Neotropica, v. 6, n. 2, 2006.

BARBOSA, D. C. A. Estratégias de germinação e crescimento de espécies lenhosas da caatinga com germinação rápida. In: LEAL, I. R; TABARELLI, M.; SILVA, J. M. C. (eds.). Ecologia e conservação da Caatinga. Recife, Ed. da Universidade Federal de Pernambuco. 2003, p. 625-656.

BASKIN, C. C.; BASKIN. J. M. Seeds: ecology, biogeography, and evolution of dormancy and germination. San Diego: Academic Press, 1998. $666 \mathrm{p}$.

BERGER, A. P. A. Variabilidade intra-específica em Lithraea molleoides (Vell.) Eng. (aroeirabranca) a partir dos processos de germinação e emergência. 2007. 57 f. Dissertação (Mestrado em Agronomia - Fitotecnia) - Universidade Federal de Uberlândia, 2007.

BERGER, A. P. A. et al. Emergência de plântulas de Myracrodruon urundeuva Allemão (Anacardiaceae) do vale do Rio Araguari, MG. Revista Brasileira de Biociências, Porto Alegre, v. 5, n. 2, p. 10291031, jul. 2007.

BORGES, K. C. F. et al. Germinação de sementes e emergência de plântulas de Luehea divaricata Mart. Revista Brasileira de Biociências, Porto Alegre, v. 5, n. 2, p. 1008-1010, jul. 2007.

BORGES, S. et al. Equilíbrio higroscópico e viabilidade de sementes de angico-vermelho (Anadenanthera peregrina (L.) Speng) em diferentes condições ambientais de armazenamento. Scientia Forestalis, Piracicaba, v. 37, n. 3, p. 475481, dez. 2009.

CAMARGO, J. L. C.; FERRAZ, I. D. K.; IMAKAWA, A. M. Rehabilitation of degraded areas of central Amazonia using direct sowing of forest tree seeds. Restoration Ecology, v. 10, n. 4, Dec. 2002.

CARDOSO, E.; SCHIAVINI, I. Relação entre distribuição de espécies arbóreas e topografia em um gradiente florestal na Estação Ecológica do Panga (Uberlândia, MG). Revista Brasileira de Botânica, São Paulo, v. 25, n. 3, p. 277-289, set. 2002.

CARVALHO, K. S.; VASCONCELOS, H. L. Forest fragmentation in central Amazonia and its effects on litter-dwelling ants. Biological Conservation, $n$. 91, p. 151-157, 1999.

CARVALHO, L. R.; SILVA, E. A. A.; DAVIDE, A. C. Classificação de sementes florestais quanto ao comportamento no armazenamento. Revista Brasileira de Sementes, v. 28, n. 2, p. 15-25, 2006. CARVALHO, M. P.; SANTANA, D. G.; RANAL, M. A. Emergência de plântulas de Anacardium humile A. St.-Hil. (Anacardiaceae) avaliada por meio de amostras pequenas. Revista Brasileira de Botânica, São Paulo, v. 28, n. 3, p. 627-633, jul./ set. 2005.

CARVALHO,P.E.R.Espéciesflorestais brasileiras: recomendações silviculturais, potencialidades e uso da madeira. Brasília: EMBRAPA. Informações Tecnológicas, Colombo, EMPRAPA-CNPF, 2003, 1039 p. v. 1.

CASTRO, R. D.; BRADFORD, K. J.; HILHORST, H. W. M. Desenvolvimento de sementes e conteúdo de água. In: FERREIRA, A. G.; BORGHETTI, F. (orgs.). Germinação: do básico ao aplicado. Porto Alegre, Artmed, 2004, p. 51-67.

COMMANDER, L. E. et al. Seed biology of Australian arid zone species: Germination of 18 species used for rehabilitation. Journal of Arid Environments, n. 73, p. 617-625, 2009.

DECKERS, B. et al. Differential environmental response of plant functional types in hedgerow 
habitats. Basic and Applied Ecology, n. 5, p. 551556, 2004.

DEMINICIS, B. B. et al. Dispersão natural de sementes: importância, classificação e sua dinâmica nas pastagens tropicais. Archivos de Zootecnia, v. 58, p. 35-58, 2009.

DE-PAULA, O. C.; OLIVEIRA, D. M. T. Multiple pleurograms in Chamaecrista Moench (Leguminosae, Caesalpinioideae). Botanical Journal of the Linnean Society, n. 157, p. 487492, 2008.

DIETZSCH, L. et al. Caracterização da flora arbórea de dois fragmentos da mata de galeria do Parque Canjerana, DF. Cerne, Lavras, v. 12, n. 3, p. 201210, jul./set. 2006.

DOBSON, A. P.; BRADSHAW, A. D.; BAKER, A. J. M. Hopes for the future: restoration ecology and conservation biology. Science, v. 277, jul. 1997.

DORNELES, M. C. Padrões de germinação dos diásporos e emergência das plântulas de espécies arbóreas do Cerrado, do Vale do Rio Araguari, MG. 2010. 126 f. Tese (Doutorado em Ecologia e Conservação de Recursos Naturais) - Universidade Federal de Uberlândia, 2010.

DORNELES, M. C.; RANAL, M. A.; SANTANA, D. G. Germinação de diásporos recém-colhidos de Myracrodruon urundeuva Allemão (Anacardiaceae) ocorrente no cerrado do Brasil Central. Revista Brasileira de Botânica, São Paulo, v. 28, n. 2, p. 399-408, abr.jun. 2005.

DURIGAN, G. Bases e diretrizes para a restauração da vegetação de cerrado. In: KAGEYAMA, P. Y.; OLIVEIRA, R. E.; MORAES, L. F. D.; ENGEL, V. L.; GANDARA, B. F. (eds.). Restauração Ecológica de Ecossistemas Naturais. Botucatu: Ed. do Lar Anália Franco/FEPAF, 2003, p. 185-204. FERREIRA, W. R. Variabilidade de cinco espécies arbóreas da região de Cerrado do Brasil central para medidas de germinação e emergência. 2007. 116 f. Dissertação (Mestrado em Agronomia - Fitotecnia) - Universidade Federal de Uberlândia, 2007.

FERRETE, J. A. et al. Ecótopos encontrados em perfis topográficos traçados nas áreas de implantação das barragens das UHEs de capim branco I e II, na bacia do rio Araguari, no município de Uberlândia (MG). Caminhos de Geografia, Uberlândia, v. 16, n. 16, p. 172-189, out. 2005.

GARCIA, L. C.; NOGUEIRA, A. C.; ABREU, D. C. A. Influência do envelhecimento acelerado no vigor de sementes de Anadenanthera colubrina (Vellozo) Brenan - Mimosaceae. Ciência Florestal,
Santa Maria, v. 14, n. 1, p. 85-90, 2004.

GARWOOD, N. C. Seed Germination in a seasonal tropical Forest in Panamá: a community study. Ecological Monographs, v. 53, n. 2, p. 159-181, 1983.

GASCON, C. et al. Matrix habitat and species richness in tropical forest remnants. Biological Conservation, n. 91, p. 223-229, 1999.

GUNN, C. R. Seeds of the Leguminosae. In: POLHILL, R. M.; RAVEN, P. H. (eds.). Advances in Legume Systematics. Kew: Ed. Crown, 1981, p. 913-926.

GUNN, C. R. Fruits and seeds of genera in the subfamily Caesalpinioideae (Fabaceae). United States: Department of Agriculture, 1991. p. 1-408, (Technical Bulletin, n. 1755)

HYDE, E. O. C. The function of the hilum in some Papilionaceae in relation to the ripening of the seed and the permeability of the testa. Annals of Botany, n. 18, p. 241-256, 1954.

KAGEYAMA, P. Y. et al. Diversidade genética em espécies árboreas tropicais de diferentes estágios sucessionais por marcadores genéticos. Scientia Forestalis, n. 4, p. 93-107, dez. 2003.

KLINK, C. A.; MACHADO, R. B. A conservação do Cerrado brasileiro. Megadiversidade, v. 1, n. 1, p. 147-155, jul. 2005.

KOLB, A.; DIEKMANN, M. Effects of lifehistory traits on responses of plant species to forest fragmentation. Conservation Biology, v. 19, n. 3, Jun. 2005.

KÖPPEN, W. Climatología: con un estudio de los climas de la Tierra. Pérez, H. P. R. (trad.). México: Fondo de Cultura Economica, 1948, p. 152-282.

LAVOREL, S. I. et al. Identifying functional groups for response to disturbance in an abandoned pasture. Acta Oecologia, v. 19, n. 3, p. 227-240, 1998.

LIMA, J. A.; SANTANA, D. G.; NAPPO, M. E. Comportamento inicial de espécies na revegetação da mata de galeria na Fazenda Mandaguari, em Indianópolis, MG. Revista Árvore, Viçosa, v. 33, n. 4, p. 685-694, 2009.

LIMA, V. V. F. et al. Germinação de espécies arbóreas de Floresta Estacional Decidual do Vale do Rio Paranã em Goiás após três tipos de armazenamento por até 15 meses. Biota Neotropical, v. 8, n. 3, p. 89-97, jul./set. 2008.

LOPES, W. P. et al. Composição da flora arbórea de um trecho de Floresta Estacional no Jardim Botânico da Universidade Federal de Viçosa (face sudoeste), Viçosa, Minas Gerais. Revista Árvore, Viçosa, v. 26, n. 3, p. 339-347, 2002. 
MENDES-RODRIGUES, C. et al. Germinação de embriões de duas espécies de Inga (Mimosaceae). Revista Brasileira de Biociências, Porto Alegre, v. 5, n. 2, p. 561-563, jul. 2007.

MENDES-RODRIGUES, C. et al. Multiple dormancy and maternal effect on Miconia ferruginata (Melastomataceae) seed germination, Serra de Caldas Novas, Goiás, Brazil. Revista Brasileira de Botânica, v. 33, n. 1, p. 93-105, jan./ mar. 2010.

MENDONÇA, R. C. et al. Flora Vascular do Bioma Cerrado: "Checklist" com 12.356 espécies. In: SANO, S. M.; ALMEIDA, S. P.; RIBEIRO, J. F. (eds.). Cerrado: Ecologia e Flora. Brasília: Embrapa Informação Tecnológica, 2008, p. 421442.

NASCIMENTO, A. R. T.; FELFILI, J. M.; MEIRELLES, E. M. Florística e estrutura da comunidade arbórea de um remanescente de Floresta Estacional Decidual de encosta, Monte Alegre, GO, Brasil. Acta Botanica Brasilica, v. 18, n. 3, p. 659-669, 2004.

PAULA, A. et al. Sucessão ecológica da vegetação arbórea em uma Floresta Estacional Semidecidual, Viçosa, MG, Brasil, Acta Botanica Brasilica, v. 18, n. 3, p. 407-423, 2004.

PAZ, H.; MARTINEZ-RAMOS, M. Seed mass and seedling performance within eight species of Psychotria (Rubiaceae). Ecology, v. 84, n. 2, p. 439-450, 2003.

PEGADO, C. M. A. et al. Efeitos da invasão biológica de algaroba - Prosopis juliflora (Sw.) DC. sobre a decomposição e a estrutura do estrato arbustivo-arbóreo da caatinga no município de Monteiro, PB, Brasil. Acta Botanica Brasilica, v. 20, n. 4, p. 887-898, 2006.

PEREIRA, R. S.; SANTANA, D. G.; RANAL, M. A. Emergência de plântulas oriundas de sementes recém-colhidas e armazenadas de Copaifera langsdorffii Desf. (Caesalpinioideae), Triângulo Mineiro, Brasil. Revista Árvore, Viçosa, v. 33, n. 4, p. 643-652, 2009.

POTT, A.; POTT, V. J.; DAMASCENO JÚNIOR, G. A. Fitogeografia do Pantanal. In: CONGRESSO DE ECOLOGIA DO BRASIL, 9, 2009, São Lourenço. Anais... Sociedade de Ecologia do Brasil: São Lourenço, 2009, p. 1-3.

RANAL, M. A.; SANTANA, D. G. How and why to measure the germination process? Revista Brasileira de Botânica, São Paulo, v. 29, n. 1, p. 1-11, jan./mar. 2006.

REGO, S. S. et al. Influência de potenciais osmóticos na germinação de sementes de Anadenanthera colubrina (Veloso) Brenan (angico-branco) Mimosaceae. Revista Brasileira de Biociências, v. 5, n. 2, p. 549-551, jul. 2007.

RODRIGUES, A. C. et al. Biometria de frutos e sementes e grau de umidade de sementes de angico (Anadenanthera colubrina (Vell.) Brenan var. cebil (Griseb.) Altschut) procedentes de duas áreas distintas. Revista Científica Eletrônica de Engenharia Florestal, Garças, v. 4, n. 8, p. 1-15, ago. 2006.

ROY, V.; BLOIS, S. Using functional traits to assess the role of hedgerow corridors as environmental filters for forest herbs. Biological Conservation, $\mathrm{n}$. 130, p. 592-603, 2006.

SALOMÃO, A. N. Tropical seed species' responses to liquid nitrogen exposure. Brazilian Journal of Plant Physiology, v. 14, n. 2, p. 133-138, 2002.

SALOMÃO, A. N. et al. Germinação de sementes e produção de mudas de plantas do cerrado. Brasília: Rede de sementes do cerrado, 2003. 96 p. SAUTU, A. et al. Studies on the seed biology of 100 native species of trees in a seasonal moist tropical forest, Panama, Central America. Forest Ecology and Management, n. 234, p. 245-263, 2006.

SILVA, A. F. et al. Composição florística e grupos ecológicos das espécies de um trecho de Floresta Semidecídua Submontana da Fazenda São Geraldo, Viçosa-MG. Revista Árvore, Viçosa, v. 27, n. 3, p. 311-319, 2003.

SIMGE. Sistema de informações meteorológicas do Estado de Minas Gerais. Disponível em: $<$ (http// www.simge.mg.gov.br) $>$ Acesso em: 10 de janeiro de 2010 .

SIQUEIRA, A. S.; ARAÚJO, G. M.; SCHIAVINI, I. Estrutura do componente arbóreo e características edáficas de dois fragmentos de floresta estacional decidual no vale do rio Araguari, MG, Brasil. Acta Botanica Brasilica, v. 23, n. 1, p. 10-21, 2009.

SOUZA, P. A. et al. Avaliação do banco de sementes contido na serapilheira de fragmento florestal visando recuperação de áreas degradadas. Cerne, Lavras, v. 12, n. 1, p. 56-67, jan./mar. 2006.

VALE, V.S. et al. Composição florística e estrutura do componente arbóreo em um remanescente primário de Floresta Estacional Semidecidual em Araguari, Minas Gerais, Brasil. Hoehnea, v. 36, n. 3, p. 299429, 2009.

VEASEY, E. A.; FREITAS, J. C. T.; SCHAMMASS, E. A. Variabilidade da dormência de sementes entre e dentro de espécies de Sesbania. Scientia Agricola, Piracicaba, v. 57, n. 2, p. 299-304, abr./jun. 2000. 
WERNECK, M. S.; FRANCESCHINELLI, E. V.; de quatro anos (1994-1998), região do Triângulo TAMEIRÃO-NETO, E. Mudanças na florística e Mineiro, MG. Revista Brasileira de Botânica, São estrutura de uma floresta decídua durante um período Paulo, v. 23, n. 4, p. 401-413, dez. 2000. 